\title{
Practical and Simple Wireless Channel Models for Use in Multipolarized Antenna Systems
}

\author{
KwangHyun Jeon, Xin Su, Bing Hui, and KyungHi Chang \\ Electronic Engineering Department, Inha University, Incheon 402-751, Republic of Korea \\ Correspondence should be addressed to KyungHi Chang; khchang@inha.ac.kr
}

Received 21 February 2014; Accepted 10 May 2014; Published 25 May 2014

Academic Editor: Paschalis Sofotasios

Copyright (C) 2014 KwangHyun Jeon et al. This is an open access article distributed under the Creative Commons Attribution License, which permits unrestricted use, distribution, and reproduction in any medium, provided the original work is properly cited.

The next-generation wireless systems are expected to support data rates of more than $100 \mathrm{Mbps}$ in outdoor environments. In order to support such large payloads, a polarized antenna may be employed as one of the candidate technologies. Recently, the third generation partnership standards bodies (3GPP/3GPP2) have defined a cross-polarized channel model in SCM-E for MIMO systems; however, this model is quite complex since it considers a great many channel-related parameters. Furthermore, the SCME channel model combines the channel coefficients of all the polarization links into one complex output, making it impossible to exploit the MIMO spatial multiplexing or diversity gains in the case of employing polarized antenna at transmitter and receiver side. In this paper, we present practical and simple 2D and 3D multipolarized multipath channel models, which take into account both the cross-polarization discrimination (XPD) and the Rician factor. After verifying the proposed channel models, the BER and PER performances and throughput using the EGC and MRC combining techniques are evaluated in multipolarized antenna systems.

\section{Introduction}

The next-generation wireless systems are required to possess high voice quality and high data rate services compared to the current cellular mobile radio standards and also provide seamless data service. Recent work has shown that independent spatial channels can be used to greatly enhance capacity under situations subject to scattering, such as urban areas or indoor environments [1-3]. Multiple input multiple output (MIMO) systems have been shown to dramatically increase the capacity of wireless systems and so have drawn an increasing amount of attention in recent years [4]. This capacity gain, however, strongly depends on the transmit $(\mathrm{Tx})$ and receive $(\mathrm{Rx})$ antenna spacing. In practical situations, antennas spacing of several wave lengths is required in order to achieve a significant multiplexing gain. Unfortunately, this large antenna spacing requirement increases both the size and the cost of base stations and makes it quite difficult to render the use of multiple antennas in customer devices. Antenna elements employing multiple polarizations can increase the capacity and often require less space per Tx and Rx channel than spatially separated polarization elements [57].

A great deal of research is in progress in regard to spatial MIMO channel models; however, models regarding the polarized antenna channel are still in their early stages. Existing polarized antenna channel models can be divided into two categories: two-dimensional (2D) channel models where all of the scatterers are located in a $2 \mathrm{D}$ plane and threedimensional (3D) channel models where all the scatterers are located in 3D space. One of the well-known 2D polarized antenna channel models is the one defined by the spatial channel model extended (SCM-E) [8], which is quite complex since it considers a great many parameters, such as angle spread, angle of arrival, angle of departure, power azimuth spectrum, lognormal shadowing, delay spread, and path loss. On top of that, there is only a single output of complex channel gain from the SCM-E polarized channel model resulting from the combination of all polarized channel links. When polarized antenna is employed at both Tx and Rx sides, 
it is not possible to exploit spatial multiplexing gain or other types of combining gains using the SCM-E polarized antenna channel model since it combines the channel coefficients of all the polarization links into one complex output. In addition, it is not convenient to observe the characteristics of each polarized channel link (e.g., $v-h, h-v, h-h$, and $v-v ; v$ for vertical, $h$ for horizontal), such as the cross-polarization discrimination (XPD) values and the contributions from the above four types of polarized links. This is because it obtains only a single complex output resulting from the combination of all polarized links. In this case, it is impossible to exploit spatial multiplexing gain or other types of combining gains since the polarized links become indistinguishable from each other. Here, XPD is defined as the ratio of the copolarized average received power to the cross-polarized average received power; it has an essential role in the polarized antenna channel models in quantifying the separation between two transmission channels that use different polarization orientations. 3D geometry-based statistical modeling of channel depolarization for polarized antenna systems has been proposed by Kwon and Stüber in [9]. However, it is also quite complicated since it also considers many parameters, such as the cylindrical scattering surface radius, the azimuth angle of arrival, the elevation angle of arrival, and the distance between the base station (BS) and mobile station (MS). Furthermore, the double integrals in [9] by assuming an infinite number of scatterers make their channel model too complex to be practically implemented. Another typical 3D polarized MIMO channel based on SCM 2D channel model is given in [10]. The spherical coordinate system is the same as our proposed scatterer structure; however, their 3D channel model is derived from SCM 2D channel model, which can be considered as update of SCM channel model in mathematical perspective. Similar problems as aforementioned about SCME channel model still exist in their 3D MIMO channel model.

In our preliminary work [11], we suggested the dualpolarized channel model mainly based on the $2 \mathrm{D}$ scattering scenario. In order to complete our research work, in this paper, a practical and simple (PS) wireless channel model for use in multipolarized (i.e., dual-/triple-polarized) antenna systems based on both $2 \mathrm{D}$ and $3 \mathrm{D}$ scattering scenarios is presented. Our proposed channel model aims at the indoor or dense urban communication environment. Therefore by referring to [2], we assume that the statistical locations of the scatterers are uniformly distributed around the MS. In addition by assuming that the energy contribution of remote scatters is negligible, a finite but sufficient number of scatterers are satisfied to reflect the scattering scenario in the proposed model. The proposed channel model preserves the continuity of time-varying phase response, which is caused by the mobility of the MS. Thus, the practical channel estimation using the training sequence becomes meaningful in the aspect of phase compensation under the proposed channel model.

The remaining parts of this paper are organized as follows. Section 2 introduces the propagation characteristics of polarized antenna systems. Section 3 presents the proposed 2D and 3D polarized channel models. The verification of the proposed polarized antenna channel models through receiver diversity is discussed in Section 4, and conclusions are drawn in Section 5.

\section{Propagation Characteristics of Polarized Antenna Systems}

This section describes the propagation characteristics of polarized antenna systems. Usually there are multiple scatterers, such as buildings, cars, and trees, uniformly located around the MS in urban environments. The propagation characteristics of polarized antenna systems can be determined through XPD, which quantifies the separation between two transmission channels that use different polarization orientations as

$$
\begin{aligned}
\mathrm{XPD} & =\frac{\text { Cochannel Rx Power }}{\text { Cross-channel Rx Power }} \\
& =\frac{\mathrm{Rx} \text { Power }(\mathrm{VV})}{\mathrm{Rx} \text { Power }(\mathrm{VH})} \text { or } \frac{\mathrm{Rx} \text { Power }(\mathrm{HH})}{\mathrm{Rx} \text { Power }(\mathrm{HV})} .
\end{aligned}
$$

The larger the XPD, the smaller the amount of energy coupled between the cross-polarized channels. When a horizontally polarized antenna receives a signal sent from a vertically polarized antenna (and vice versa), the received signal strength is reduced in proportion to the XPD value. $\mathrm{XPD}$ values have been found to decrease with an increase in the distance [9]. Usually, the XPD is revealed to have the relation with the antenna properties, distance between the transmitter and receiver, scatterers distributions, and so forth. However, in this paper, we defined XPD as the ratio of the copolarized average received power to the crosspolarized average received power [9], where it is specified only by the power coupled to the polarized channel links. In order to minimize the modeling complexity without loss of accuracy, we directly assign the XPD value according to the measurement results returned by literature references, for example, $5.8 \mathrm{~dB}$ in [9].

For polarized channel modeling in macro and micro cell environments, we use the same number of scatterers, six, as laid out in the 1st and 2nd mid rays of the SCME urban channel model [8]. Two kinds of channel models are introduced in this paper, based on the channel model components, one of which contains only non-line-of-sight (NLoS) components and the other both line-of-sight (LoS) and NLoS components, that is, the Rayleigh channel model and the Rician channel model, respectively.

2.1. The 2D Scatterer Model. As shown in Figure 1, the MS mobility is taken into consideration in regard to the polarized antenna channel model found in [11]. $\theta$ is the phase rotation of the received signal at the MS due to the scatterers, and $\theta^{\prime}$ is the updated phase rotation according to the MS movement, as described by

$$
\theta^{\prime}=\arctan \left(\frac{r \sin \theta}{r \cos \theta+\Delta}\right),
$$




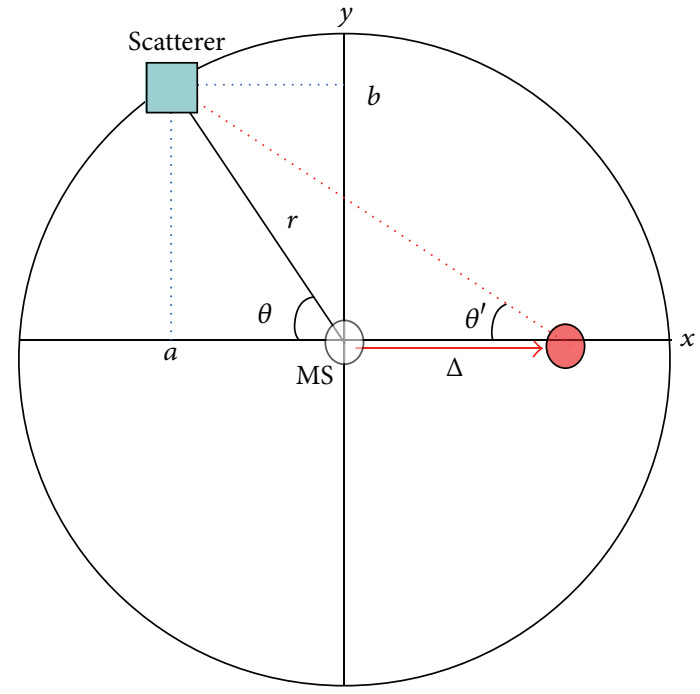

FIGURE 1: 2D scatterer model.

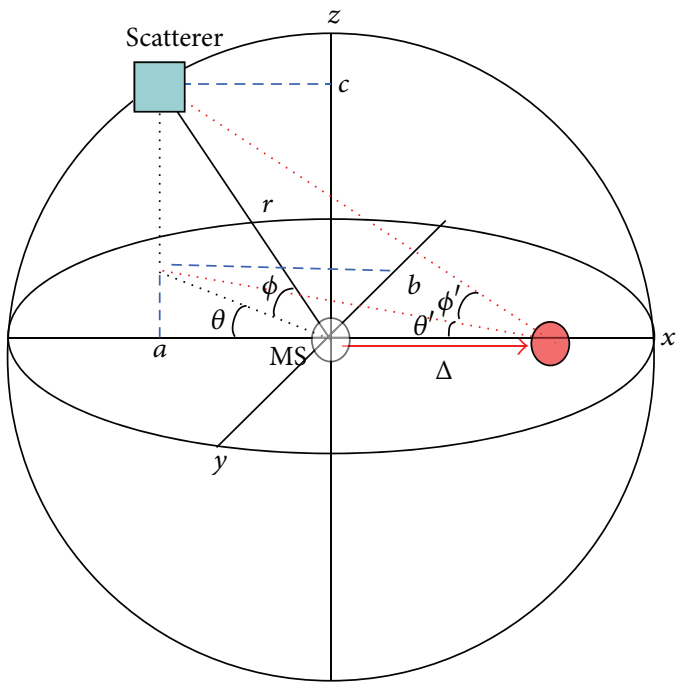

Figure 2: 3D scatterer model. where $r$ is the radius of the scatterers and $\Delta$ is the moving distance of the MS for a 1-packet duration (e.g., 5 us). In this case, $\Delta$ is quite a small value compared to the radius $r$, so we assume that the moving direction of the MS does not change within such a short time duration. This assumption will be explained in more detail when introducing the system parameters described in Section 4 . The details of the parameters for the $2 \mathrm{D}$ scatterer model are presented in Table 1.

2.2. The $3 D$ Scatterer Model. We extend the $2 \mathrm{D}$ scatterer model to the $3 \mathrm{D}$ scatterer model by adding a new parameter, $\phi$, which is the elevation angle, as shown in Figure 2. Similar to the $2 \mathrm{D}$ scatter model, the elevation angle $\phi$ represents the phase rotation of the received signal projected in the $x-z$ plane (due to the movement of the MS along the $x$ axis); $\phi^{\prime}$ is the updated phase rotation projected in the $x-z$ plane according to the MS movement. The azimuth and the elevation angles related to the phase rotations seen above are obtained by using the triangular function as shown in (3) and (4), respectively. The parameters used for the 3D scatterer model are described in Table 2. Consider

$$
\begin{aligned}
\theta^{\prime} & =\arctan \left(\frac{b}{a+\Delta}\right) \\
\phi^{\prime} & =\arctan \left(\frac{c}{a+\Delta}\right) .
\end{aligned}
$$

2.3. The Propagation Characteristics of the Rician Channel. The analytical performance under Rician polarized channel environment was discussed in [12]. The polarization matrix $\mathbf{H}$ in (5) includes the degree of suppression of the individual co/cross-polarized components, the cross-correlation, and the
TABLE 1: 2D scatterer model parameters.

\begin{tabular}{ll}
\hline$a$ & Scatterer signal projected to $x$-axis $(a=r \cos \theta)$ \\
$b$ & Scatterer signal projected to $y$-axis $(b=r \sin \theta)$ \\
$r$ & Distance between scatterers and MS \\
$\theta$ & Phase rotation of received signal at MS \\
$\theta^{\prime}$ & Updated phase rotation according to MS movement \\
$\Delta$ & Moving distance of MS \\
\hline
\end{tabular}

\begin{tabular}{|c|c|}
\hline$a$ & Scatterer signal projected to $x$-axis $(a=r \cos \theta \cos \phi)$ \\
\hline$b$ & Scatterer signal projected to $y$-axis $(b=r \cos \theta \sin \phi)$ \\
\hline$c$ & Scatterer signal projected to $z$-axis $(c=r \sin \theta)$ \\
\hline$r$ & Distance between scatterers and MS \\
\hline$\theta$ & $\begin{array}{l}\text { Phase rotation of received signal projected in } x-y \text { plan } \\
\text { at MS }\end{array}$ \\
\hline$\theta^{\prime}$ & $\begin{array}{l}\text { Updated phase rotation projected in } x-y \text { plan according } \\
\text { to MS movement }\end{array}$ \\
\hline$\phi$ & $\begin{array}{l}\text { Phase rotation of received signal projected in } x \text { - } z \text { plan } \\
\text { at MS }\end{array}$ \\
\hline$\phi^{\prime}$ & $\begin{array}{l}\text { Updated phase rotation projected in } x-z \text { plan according } \\
\text { to MS movement }\end{array}$ \\
\hline$\Delta$ & Moving distance of MS \\
\hline
\end{tabular}

TABLE 2: 3D scatterer model parameters.

cross-coupling of the energy from one polarization state to the other polarization state. Consider

$$
\mathbf{H}=\left[\begin{array}{ll}
h_{v, v} & h_{v, h} \\
h_{h, v} & h_{h, h}
\end{array}\right] .
$$

The elements of polarized channel matrix $\mathbf{H}$ are, in general, correlated to complex Gaussian random variables. 
We decompose the channel matrix into the sum of an average (or fixed, possibly LoS) component and a variable (or scattered) component using

$$
\mathbf{H}=\sqrt{\frac{k}{k+1}} \cdot \overline{\mathbf{H}}+\sqrt{\frac{1}{k+1}} \cdot \widetilde{\mathbf{H}},
$$

where $k$ is the Rician factor, $\overline{\mathbf{H}}$ is the LoS component, and $\widetilde{\mathbf{H}}$ is the NLoS component. Here, $\varepsilon\{\mathbf{H}\}=\sqrt{k /(1+k)} \cdot \overline{\mathbf{H}}$ and $\sqrt{1 /(1+k)} \cdot \widetilde{\mathbf{H}}$ are the average and variable components of the channel matrix, respectively. The factors $\sqrt{k /(1+k)}$ and $\sqrt{1 /(1+k)}$ in (6) are the power normalization factors. The elements of matrix $\widetilde{\mathbf{H}}$ are denoted as $\widetilde{h}_{v, h}(v, h: x$-pol component). The signal power of the NLoS components is determined using

$$
\begin{aligned}
& \varepsilon\left\{\left|\widetilde{h}_{h, h}\right|^{2}\right\}=\varepsilon\left\{\left|\widetilde{h}_{v, v}\right|^{2}\right\}=1, \\
& \varepsilon\left\{\left|\widetilde{h}_{h, v}\right|^{2}\right\}=\varepsilon\left\{\left|\widetilde{h}_{v, h}\right|^{2}\right\}=\alpha,
\end{aligned}
$$

where $0 \leq \alpha \leq 1$ is related to the XPD value for the variable components of the channel. A good discrimination of the orthogonal polarizations has a small XPD value, and vice versa. The elements of matrix $\overline{\mathbf{H}}$, which are denoted as $\bar{h}_{v, h}$, are non-time-varying complex numbers satisfying

$$
\left|\bar{h}_{h, h}\right|=\left|\bar{h}_{v, v}\right|=1, \quad\left|\bar{h}_{h, v}\right|=\left|\bar{h}_{v, h}\right|=\alpha_{f},
$$

where $0 \leq \alpha_{f} \leq 1$ is directly related to the XPD value for the fixed component of the channel. It is important to note that the presence of a fixed channel component does not always imply LoS conditions. For pure LoS conditions, $\alpha_{f}$, unlike $\alpha$, is solely a function of the antenna's ability to separate the orthogonal polarizations. The XPD values presented in $\mathrm{dB}$ and in linear values are related to $\alpha$ as shown by

$$
\mathrm{XPD}(\mathrm{dB})=10 \log \left(\frac{1}{\sqrt{\alpha}}\right)^{2}, \quad \mathrm{XPD}=\frac{1}{\alpha}
$$

The Rician factor for a fading channel is defined as the ratio of the power in the fixed component to the power in the variable components. Under the definitions of $\alpha$ and $\alpha_{f}$ made above, the Rician factor for each element of the channel matrix can be expressed as

$$
\begin{aligned}
& \mathbf{K}=\left[\begin{array}{ll}
k_{v, v} & k_{v, h} \\
k_{h, v} & k_{h, h}
\end{array}\right]=\left[\begin{array}{cc}
k & \frac{\alpha_{f}}{\alpha} k \\
\frac{\alpha_{f}}{\alpha} k & k
\end{array}\right] \\
& h_{u, s, n}(t)=\sqrt{\frac{P_{n} \sigma_{\mathrm{SF}}}{M}} \sum_{m=1}^{M}\left(\left[\begin{array}{c}
\chi_{\mathrm{BS}}^{(v)}\left(\theta_{n, m, \mathrm{AoD}}\right) \\
\chi_{\mathrm{BS}}^{(h)}\left(\theta_{n, m, \mathrm{AoD}}\right)
\end{array}\right]^{T}\left[\begin{array}{cc}
\exp \left(j \Phi_{n, m}^{(v, v)}\right) & \sqrt{r_{n 1}} \exp \left(j \Phi_{n, m}^{(h, v)}\right) \\
\sqrt{r_{n 2}} \exp \left(j \Phi_{n, m}^{(v, h)}\right) & \exp \left(j \Phi_{n, m}^{(h, h)}\right)
\end{array}\right]\left[\begin{array}{c}
\chi_{\mathrm{MS}}^{(v)}\left(\theta_{n, m, \mathrm{AoA}}\right) \\
\chi_{\mathrm{MS}}^{(h)}\left(\theta_{n, m, \mathrm{AoA}}\right)
\end{array}\right]\right. \\
& \left.\times \exp \left(j k d_{s} \sin \left(\theta_{n, m, \mathrm{AoD}}\right)\right) \times \exp \left(j k d_{u} \sin \left(\theta_{n, m, \mathrm{AoA}}\right)\right) \times \exp \left(j k\|v\| \cos \left(\theta_{n, m, \mathrm{AoA}}-\theta_{v}\right) t\right)\right)
\end{aligned}
$$

$\mathbf{H}_{2 \text { D-Rayleigh }}(t)$

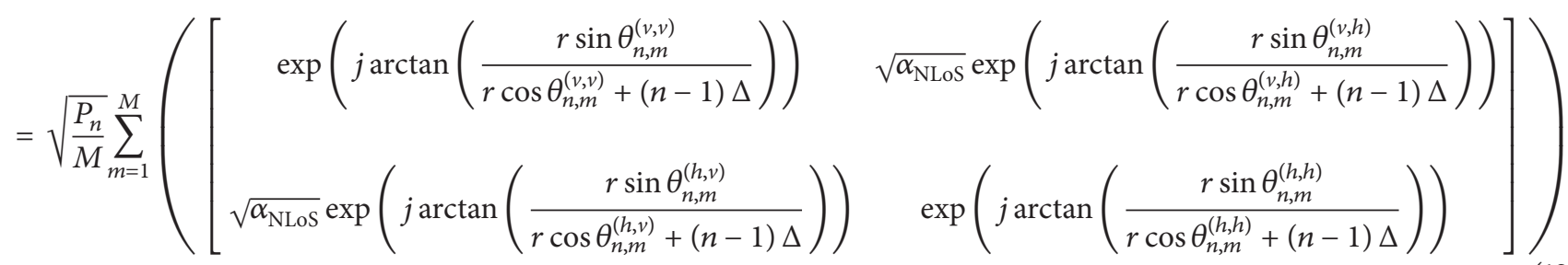

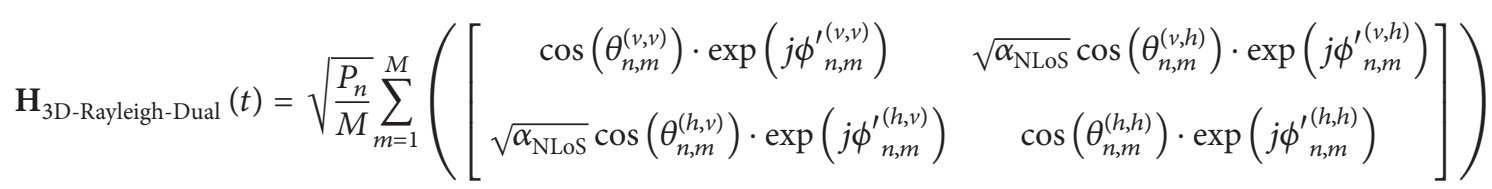




$$
\begin{aligned}
& \mathbf{H}_{3 \text { D-Rayleigh-Triple }}(t)
\end{aligned}
$$

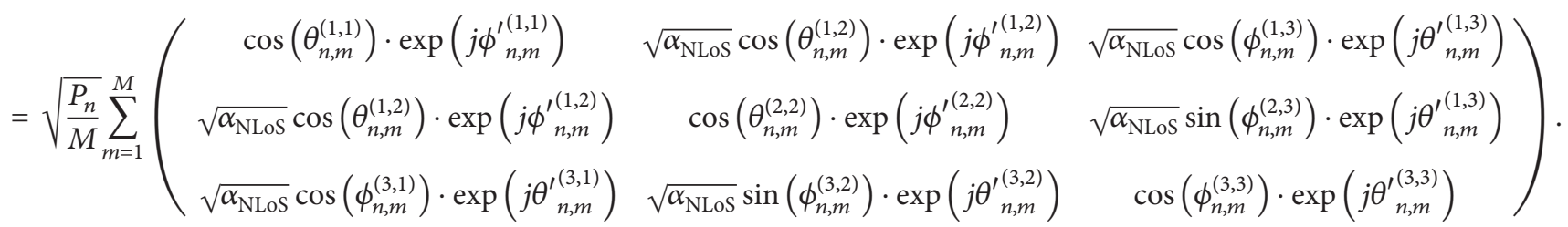

\section{Proposed Practical and Simple Multipolarized Channel Models}

The practical and simple (PS) multipolarized channel models are presented in this section; they are categorized into the Rayleigh channel model and the Rician channel model through the consideration of the wireless channel statistical properties. In addition, according to the scatterer environments, they are further classified into a 2D channel model and a 3D channel model.

The PS multipolarized channel models are based on SCME channel model given by (11). It is a well-known 2D PMIMO channel model, which obtains only a single complex output resulting from the combination of all the polarized links. In this case, it is impossible to exploit spatial multiplexing gain or other types of combining gains since the polarized links become indistinguishable from each other. Here, $P_{n}$ is the power of the $n$th path, $\sigma_{\mathrm{SF}}$ is the lognormal shadow fading, and $M$ is the number of subpaths per path. $\theta_{n, m, \mathrm{AoD}}$ is the AoD for the $m$ th subpath of the $n$th path, and $\theta_{n, m, \mathrm{AoA}}$ is the AoA for the $m$ th subpath of the $n$th path. $k$ is the wave number, $d_{s}$ is the distance in meters between the BS antenna elements and the reference antenna element in the BS antenna array, and $d_{u}$ is the distance in meters between the MS antenna elements and the reference antenna element in the MS antenna array. The SCM-E channel model defines $\Phi_{n, m}^{(x, y)}$ to be the phase offset of the $m$ th subpath of the $n$th path between the $x$ component (e.g., either the horizontal $h$ or vertical $v$ ) of the BS element and the $y$ component (e.g., either the horizontal $h$ or vertical $v$ ) of the MS element as i.i.d. random variables drawn from a uniform 0- to 360degree distribution. $x$ and $y$ can alternatively represent the copolarized and cross-polarized orientations. $\|v\|$ and $\theta_{v}$ are the magnitude and angle of the MS velocity vector, respectively. $\chi_{\mathrm{BS}}^{(x)}\left(\theta_{n, m, \mathrm{AoD}}\right)$ and $\chi_{\mathrm{MS}}^{(x)}\left(\theta_{n, m, \mathrm{AoA}}\right)$ are the BS and MS antenna complex response for the $x$-pol component, respectively. $r_{n 1}$ and $r_{n 2}$ are the linear XPD values in the SCME model, as described in [8].

The SCM-E channel model is very complex for use in imitating the practical wireless propagation channel since it employs a pseudoray tracing model. Another drawback is that there is only a single output of complex channel gain from the SCM-E polarized channel model for each instant in time, which makes the exploiting of MIMO spatial multiplexing or Rx combining techniques impossible. Moreover, it is not convenient to observe the characteristics of each of the polarized channel links (e.g., $v-h, h-v, h-h$, and $v-v$ ), such as the XPD values and contributions from the above four polarized links. Therefore, we develop new channel models that use a matrix determining the channel complex gain and thereby represent the characteristics of all the polarized channel links, that is, $v-h, h-v, h-h$, and $v-v$.

3.1. The PS 2D Dual-Polarized Rayleigh Channel Model. PS 2D dual-polarized Rayleigh channel is modeled as depicted by (12) based on the polarized characteristics of the SCME channel model applied to the 2D scatterer environment shown in Figure 1. Comparisons between the proposed channel model and the SCM-E channel model are given through four perspectives below.

Angle between Antenna Branches. $\chi_{\mathrm{BS}}^{(x)}\left(\theta_{n, m, \mathrm{AoD}}\right)$ and $\chi_{\mathrm{MS}}^{(x)}$ $\left(\theta_{n, m, \mathrm{AoA}}\right)$ in (11) describe the angle between the $x$-pol antenna branches in the SCM-E channel model. In our proposed channel model, however, we assume that the $v$ and $h$ branches at $\mathrm{Tx}$ (or $\mathrm{Rx}$ ) are always orthogonal with respect to each other. That is, only the polarized channel characteristics are considered in our channel model. So, the parameters, such as $\chi_{\mathrm{BS}}^{(x)}\left(\theta_{n, m, \mathrm{AoD}}\right)$ and $\chi_{\mathrm{MS}}^{(x)}\left(\theta_{n, m, \mathrm{AoA}}\right)$, used to reflect the angle between the $v$ and $h$ branches can be considered additionally when the elliptic polarized antenna is assumed or the slant angel mismatch exists for $\mathrm{Tx}$ and $\mathrm{Rx}$ polarized antennas.

Fading Channel. In our proposed channel model, as in the SCM-E polarized channel model, $\theta_{n, m}^{(v, h)}$ is the phase offset of the $m$ th subpath of the $n$th path, which are i.i.d. random variables drawn from a uniform 0- to 360-degree distribution. The phase rotation in the SCM-E channel model is randomly generated in a statistical sense. In order to guarantee a continuously time-varying phase response in our channel model, however, the phase offset is updated according to the MS mobility in place of random generation.

Mobility. $\exp \left(j k\|v\| \cos \left(\theta_{n, m, \mathrm{AoA}}-\theta_{v}\right) t\right)$ is a variable related to the mobility in the SCM-E channel model. However, the mobility in our proposed model is derived using $\Delta$ and $\theta$ in the channel matrix seen in (12) in order to generate the timevarying phase response. Because the mobility introduces frequency (phase) offset, by employing the parameters $\Delta$ and $\theta$, the Doppler effect can be represented intuitively. That is, the angel $\theta$ reflects the direction of mobility, and the displacement of UE within one slot period is represented by $\Delta$. The value of $\Delta$ increases as the mobility increases, so the frequency (phase) offset is also increasing. 
MIMO. $\exp \left(j k d_{s} \sin \left(\theta_{n, m, \mathrm{AoD}}\right)\right)$ and $\exp \left(j k d_{u} \sin \left(\theta_{n, m, \mathrm{AoA}}\right)\right)$ in the SCM-E channel model are variables related to the AoD and AoA of the MIMO polarized antenna system. In this study, we decide to model the SISO polarized channel to be employed in the polarized antenna system for SISO and independent MIMO cases. For the correlated MIMO polarized antenna system, the correlation regarding the SISO polarized antenna needs to be additionally considered. Furthermore, due to the assumption that there is sufficient distance between the BS and MS in our channel models, all the input signals are incident in parallel at the MS, which means that the AoD value becomes 0 in our channel models. However, the AoA angels are considered due to the scatterer environment shown in Figure 1, which contributes to the phase response of the received signal.

Instead of the independent consideration of the channel parameters used in the SCM-E channel model, all the contributions mentioned above are jointly coupled in order to build a complete channel matrix in our proposed polarized channel model, which results in the simple and practical channel model.

Based on these principles, the PS 2D dual-polarized Rayleigh channel is modeled as described in (12). Here, $M$ is the number of subpaths (scatterers), $P_{n}$ is the power of the $n$th path, $\theta_{n, m}^{(v, h)}$ is the phase rotation of the received signal at the MS from the $m$ th subpath of the $n$th path, $\alpha_{\mathrm{NLoS}}$ is the linear XPD value for the NLoS components, $r$ is the distance between the scatterers and the MS, and $\Delta$ is the moving distance of the MS. $\sqrt{P_{n} / M}$ is the power normalization factor. When a horizontally polarized antenna receives a signal sent from a vertically polarized antenna (and vice versa), the received signal strength is reduced proportionally to the linear XPD value $\alpha_{\mathrm{NLoS}}$.

\subsection{The PS 3D Dual-Polarized Rayleigh Channel Model.} The PS 3D dual-polarized Rayleigh channel is modeled as described in (13) based on the polarized characteristics of the SCM-E channel model applied to the 3D scatterer environment shown in Figure 2.

We expand the $2 \mathrm{D}$ scatterer model to the $3 \mathrm{D}$ scatterer model by adding an elevation angle parameter $\phi$ which is updated according to the MS mobility using

$$
\phi^{\prime}=\arctan \left(\frac{r \cos \theta_{n, m} \sin \phi_{n, m}}{r \cos \theta_{n, m} \cos \phi_{n, m}+(n-1) \Delta}\right) .
$$

Since a dual-polarized antenna on the $x-y$ plane is employed in the $3 \mathrm{D}$ environment, the projection of the reflected signals by the $3 \mathrm{D}$ scatterers in the $x-y$ plane is considered as seen in (13). Therefore, the 3D dual-polarized channel model depicts the channel complex gain using a $(2 \times 2)$ matrix. Similar to the $2 \mathrm{D}$ channel model, the crosschannel complex gain is attenuated by the linear XPD value $\alpha_{\mathrm{NLOS}}$.

3.3. The PS 3D Triple-Polarized Rayleigh Channel Model. The PS 3D triple-polarized Rayleigh channel is modeled as seen in (14), based on the PS 3D dual-polarized channel model.
In addition to the projected signal in the $x-y$ plane due to the dual-polarization, the projected signals in the $x-z$ and $y-z$ planes are considered in order to account for a triple polarization. The updated azimuth angle and elevation angle due to mobility are shown in (16) and (17), respectively. Consider

$$
\begin{aligned}
& \theta^{\prime}=\arctan \left(\frac{r \cos \theta_{n, m} \sin \phi_{n, m}}{r \cos \theta_{n, m} \cos \phi_{n, m}+(n-1) \Delta}\right), \\
& \phi^{\prime}=\arctan \left(\frac{r \sin \theta_{n, m}}{r \cos \theta_{n, m} \cos \phi_{n, m}+(n-1) \Delta}\right) .
\end{aligned}
$$

This channel model generates a $(3 \times 3)$ matrix for its complex channel output; the complex gain of the cross-channels is attenuated by the linear XPD value $\alpha_{\mathrm{NLoS}}$. Since the XPD values describe the long-term characteristics (primarily mean values), all of the XPD values are assumed to be the same.

3.4. The PS Multipolarized Rician Channel Model. Similar to the Rayleigh channel model described above, the PS dualpolarized Rician channel for both the $2 \mathrm{D}$ and $3 \mathrm{D}$ cases can be generated by introducing the Rician factor [12] into the Rayleigh channel model for both the LoS component and the NLoS components:

$$
\begin{aligned}
& \mathbf{H}_{\text {Rician-Dual }}(t) \\
& =\left[\begin{array}{ll}
\sqrt{\frac{k}{k+1}} & \sqrt{\frac{k^{\prime}}{k^{\prime}+1}} \\
\sqrt{\frac{k^{\prime}}{k^{\prime}+1}} & \sqrt{\frac{k}{k+1}}
\end{array}\right] \odot \overline{\mathbf{H}}(t) \\
& +\left[\begin{array}{ll}
\sqrt{\frac{1}{k+1}} & \sqrt{\frac{1}{k^{\prime}+1}} \\
\sqrt{\frac{1}{k^{\prime}+1}} & \sqrt{\frac{1}{k+1}}
\end{array}\right] \odot \mathbf{H}_{\text {Rayleigh-Dual }}(t),
\end{aligned}
$$

where $k$ is the conventional Rician factor, $k^{\prime}$ is $\left(\alpha_{\mathrm{LoS}} / \alpha_{\mathrm{NLoS}}\right) k$, and $\odot$ is the Hadamard product. The LoS component $\overline{\mathbf{H}}(t)$ is given as described by (19), and the NLoS component $\mathbf{H}_{\text {Rayleigh-Dual }}(t)$ is given as described in (12) for the $2 \mathrm{D}$ case and in (13) for the 3D case. Consider

$$
\overline{\mathbf{H}}(t)=\left[\begin{array}{cc}
\bar{h}_{v, v} & \sqrt{\alpha_{\mathrm{LoS}}} \cdot \bar{h}_{v, h} \\
\sqrt{\alpha_{\mathrm{LoS}}} \cdot \bar{h}_{h, v} & \bar{h}_{h, h}
\end{array}\right] .
$$

The dual-polarized case is extended into a $(3 \times 3)$ channel matrix for the PS 3D triple-polarized Rician channel model. Similar to the dual-polarized Rician channel model, the PS 3D triple-polarized Rician channel model is determined by the LoS component and NLoS components, as shown in (20). 
The LoS component $\overline{\mathbf{H}}(t)$ is determined by (21), and the NLoS component $\mathbf{H}_{3 \mathrm{D} \text {-Rayleigh-Triple }}(t)$ is as shown in (14):

$$
\begin{aligned}
& \mathbf{H}_{3 \text { D-Rician-Triple }}(t) \\
& =\left[\begin{array}{lll}
\sqrt{\frac{k}{k+1}} & \sqrt{\frac{k^{\prime}}{k^{\prime}+1}} & \sqrt{\frac{k^{\prime}}{k^{\prime}+1}} \\
\sqrt{\frac{k^{\prime}}{k^{\prime}+1}} & \sqrt{\frac{k}{k+1}} & \sqrt{\frac{k^{\prime}}{k^{\prime}+1}} \\
\sqrt{\frac{k^{\prime}}{k^{\prime}+1}} & \sqrt{\frac{k^{\prime}}{k^{\prime}+1}} & \sqrt{\frac{k}{k+1}}
\end{array}\right] \odot \overline{\mathbf{H}}(t) \\
& +\left[\begin{array}{ccc}
\sqrt{\frac{1}{k+1}} & \sqrt{\frac{1}{k^{\prime}+1}} & \sqrt{\frac{1}{k^{\prime}+1}} \\
\sqrt{\frac{1}{k^{\prime}+1}} & \sqrt{\frac{1}{k+1}} & \sqrt{\frac{1}{k^{\prime}+1}} \\
\sqrt{\frac{1}{k^{\prime}+1}} & \sqrt{\frac{1}{k^{\prime}+1}} & \sqrt{\frac{1}{k+1}}
\end{array}\right] \\
& \odot \mathbf{H}_{3 \mathrm{D}-\text { Rayleigh-Triple }}(t) \text {, } \\
& \overline{\mathbf{H}}(t)=\left[\begin{array}{ccc}
\bar{h}_{1,1} & \sqrt{\alpha_{\mathrm{LoS}}} \cdot \bar{h}_{1,2} & \sqrt{\alpha_{\mathrm{LoS}}} \cdot \bar{h}_{1,3} \\
\sqrt{\alpha_{\mathrm{LoS}}} \cdot \bar{h}_{2,1} & \bar{h}_{2,2} & \sqrt{\alpha_{\mathrm{LoS}}} \cdot \bar{h}_{2,3} \\
\sqrt{\alpha_{\mathrm{LoS}}} \cdot \bar{h}_{3,1} & \sqrt{\alpha_{\mathrm{LoS}}} \cdot \bar{h}_{3,2} & \bar{h}_{3,3}
\end{array}\right] .
\end{aligned}
$$

3.5. The PS Multipolarized Multipath Channel Model. The PS multipolarized channel models are designed for use in macro and micro cell environments. Therefore, the power delay profile of ITU-R M.1224 Veh. A from [13] is adopted in order to generate multipath channel models, as shown in (22). Consider

$$
\mathbf{H}_{\text {multipath }}(t)=\sum_{l=1}^{L} \sqrt{\frac{P_{l}}{M}} \mathbf{H}_{l}(t) \delta\left(t-\tau_{l}\right) \text {, }
$$

where $L$ is the number of multipaths, $P_{l}$ is the signal power per path, $\mathbf{H}_{l}$ is the polarized flat fading channel, and $\tau_{l}$ is the time delay of each path.

\section{Performance Gain by the Polarized Antenna System}

4.1. The General Parameters of Multipolarized Antenna System. The multipolarized antenna system is designed to be used in macro and micro cell environments; therefore we use 64 scatterers by setting the scatterer radius to $10 \mathrm{~m}$ and the MS mobility to 0,30 , and $60 \mathrm{~km} / \mathrm{h}$. The carrier frequency is set to $1.8 \mathrm{GHz}$ in order to use the measured XPD values found in [14]. Since the measured XPD values are adopted in the simulations, the depolarization effects due to both channel and the slant angel mismatch of polarized antenna branches are involved, which makes the simulation results more meaningful to reflect the scenario of real word. The Rician factor is chosen to be $9 \mathrm{~dB}$, which is a typical value in urban terrestrial wireless environments. All the parameters are given in Table 3.

The system bandwidth is $20 \mathrm{MHz}$, resulting in a symbol duration of $0.05 \mu \mathrm{s}$. In order to verify the proposed polarized channel model, a simple single-carrier packet structure composed of 100 QPSK symbols is applied to the multipolarized antenna system, wherein $5 \%$ of the symbols are used as pilots. When the MS mobility is $60 \mathrm{~km} / \mathrm{h}$, the MS moving distance becomes $8.335 \times 10^{-6} \mathrm{~m}$ during a 1-packet duration, which is quite a small value compared to the scatterer radius. Therefore, we can assume that the MS moving direction does not appreciably change within a 1-packet duration, as mentioned in Section 2.

4.2. The Verification of PS 3D Triple-Polarized Rayleigh Channel Model. Figure 3 shows the normalized fading envelopes of the co-/cross-channel links in the PS 3D triple-polarized Rayleigh channel model, assuming that all of the generated channel links are independent. The solid $H_{11}$ line represents the fading envelope of the cochannel link; the $H_{12}$ and $H_{13}$ dotted lines depict the envelopes of the cross-channel links. The mean value of the fading envelope for cochannel $H_{11}$ is almost $5.8 \mathrm{~dB}$ higher than those found for cross-channels $H_{12}$ and $H_{13}$ due to the NLoS XPD value shown in Table 3. Figure 3(a) shows the MS mobility at $0 \mathrm{~km} / \mathrm{h}$, wherein the channel envelopes do not change during one-packet duration (100 symbols). Figure 3(b) shows the MS mobility at $60 \mathrm{~km} / \mathrm{h}$; the channel envelopes are continuously time-varying due to the MS movement.

Figure 4 shows the phase response of the PS 3D triplepolarized channel model, which is continuously time-varying and changes about 0.002 radians during a 1-packet duration.

Figure 5 shows the power spectrum density (PSD) of the PS 3D triple-polarized channel model. The lines are designated by circles, squares, and triangles, which correspond to the MS mobility PSD at $3 \mathrm{~km} / \mathrm{h}, 30 \mathrm{~km} / \mathrm{h}$, and $60 \mathrm{~km} / \mathrm{h}$, respectively. The PSD results show that the maximum Doppler spreads are 10, 100, and $200 \mathrm{~Hz}$, respectively, according to mobility.

\subsection{The Receiver Diversity Gain under PS 3D Multipolarized} Rician Channel. Equal gain combining (EGC) and maximum ratio combining (MRC) are performed at the MS in order to combine the signals of the polarized channel links $[15,16]$. The EGC and MRC mathematical representations are given in (23) and (24), respectively, for a dual-polarized antenna. Consider

$$
\begin{aligned}
\mathrm{EGC} & =\frac{S_{v v}+S_{v h}+S_{h v}+S_{h h}}{h_{v v}+h_{v h}+h_{h v}+h_{h h}}, \\
\mathrm{MRC} & =\frac{h_{v v}^{*} \cdot S_{v v}+h_{v h}^{*} \cdot S_{v h}+h_{h v}^{*} \cdot S_{h v}+h_{h h}^{*} \cdot S_{h h}}{\left|h_{v v}\right|^{2}+\left|h_{v h}\right|^{2}+\left|h_{h v}\right|^{2}+\left|h_{h h}\right|^{2}},
\end{aligned}
$$

where $S_{v h}$ is the Tx signal power, $h_{v h}$ is the channel link, and $h_{v h}^{*}$ is the conjugate of $h_{v h}$. 


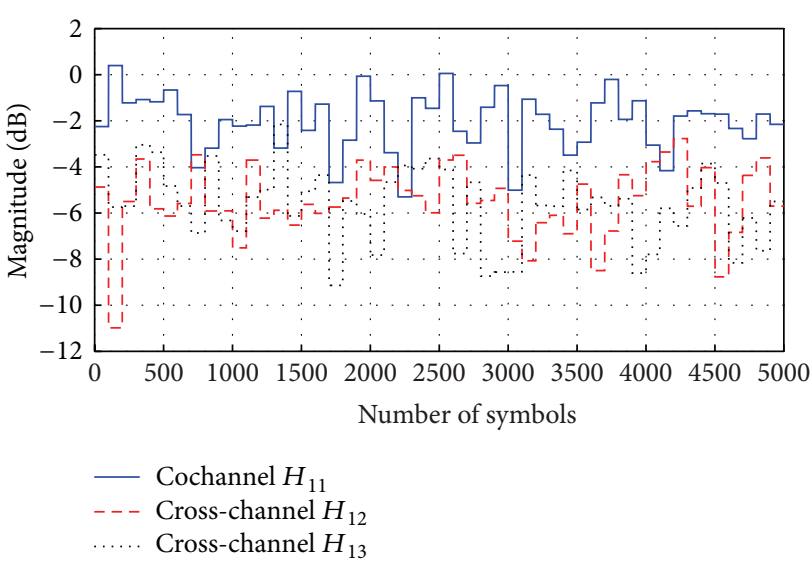

(a)

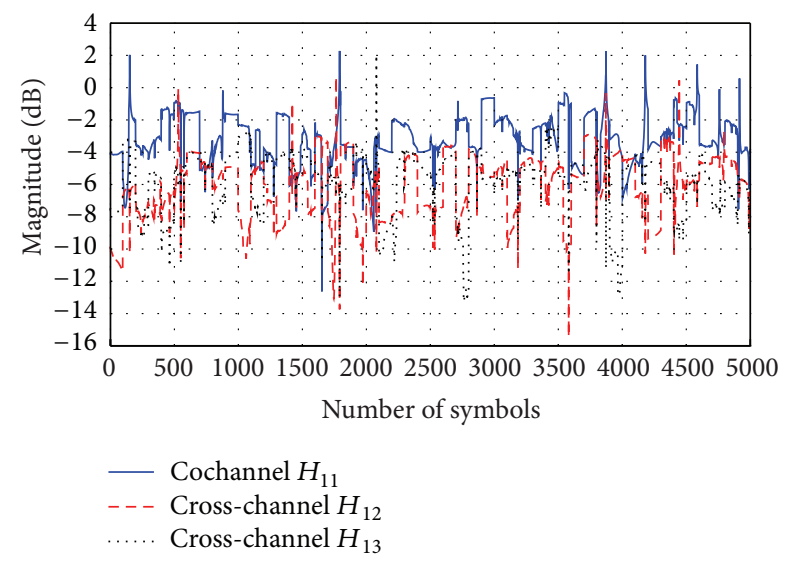

(b)

FIGURE 3: Normalized fading envelope of the PS 3D triple-polarized channel model: (a) MS mobility $=0 \mathrm{~km} / \mathrm{h}$ and (b) MS mobility $=60 \mathrm{~km} / \mathrm{h}$.

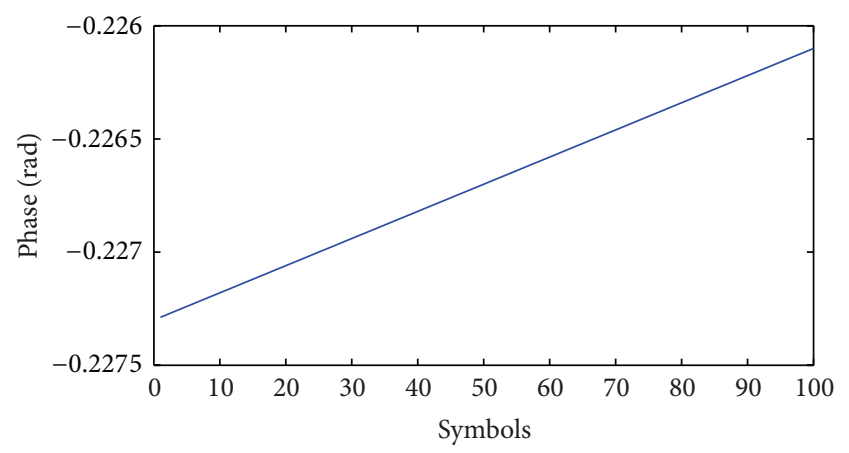

FIGURE 4: Phase response of the PS 3D triple-polarized channel model.

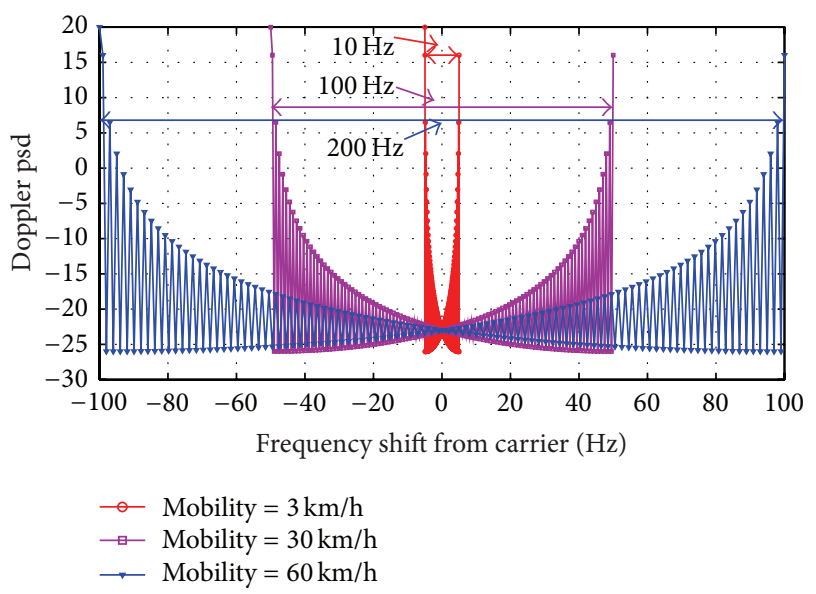

FIGURE 5: PSD of the PS 3D triple-polarized channel model.

We vary the XPD values in order to verify the proposed PS $3 \mathrm{D}$ polarized channel model in the aspect of the Eb/N0 versus bit-error-rate (BER) performance shown in Figure 6. The simulation scenario designated as "Single" shows that only a vertical dipole antenna is placed at the BS and MS.

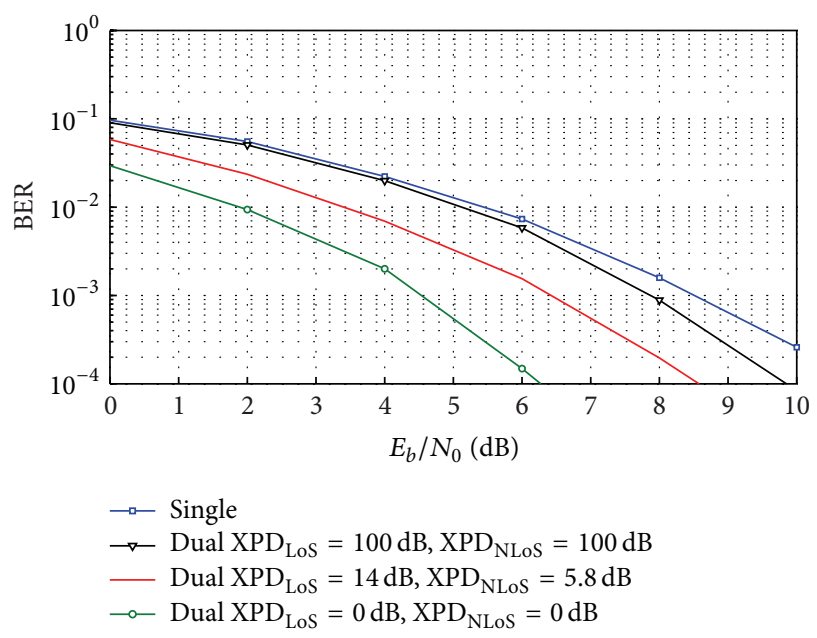

FIGURE 6: The BER performances for antennas and XPD values under a flat PS 3D fading Rician channel.

TABLE 3: System parameters.

\begin{tabular}{lc}
\hline System parameters & Values \\
\hline Target cell & Macro, micro \\
Carrier frequency & $1.8 \mathrm{GHz}$ \\
System BW & $20 \mathrm{MHz}$ \\
Scatterer radius & $10 \mathrm{~m}$ \\
Rician factor & $9 \mathrm{~dB}$ \\
LoS XPD value & $14 \mathrm{~dB}$ \\
NLoS XPD value & $5.8 \mathrm{~dB}$ \\
Number of symbols in packet & 100 symbols \\
Modulation & QPSK \\
Combining techniques & EGC, MRC \\
Equalizer & 1-tap time-domain equalizer \\
\hline
\end{tabular}


The BER performance in this case is the same as that found for a conventional SISO channel; it is used for a reference curve. The "Dual" simulation scenarios use dual-polarized antennas at the BS and MS to communicate with each other; EGC is employed to combine the signals of $v$ and $h$ links. The XPD values of $100 \mathrm{~dB}$ and $0 \mathrm{~dB}$ represent the upper and lower bounds of the BER for the dual-polarized antennas. The simulation results with XPD values of $0 \mathrm{~dB}$ achieve the best BER performance. In this case, a maximum diversity gain is obtained due to the full contributions from four channel links $(v-h, h-v, h-h$, and $v-v)$. Conversely, in the case of large $100 \mathrm{~dB}$ XPD values, the contributions from the $v-h$ and $h-v$ crosschannel links become very small compared to the $v-v$ and $h$ $h$ cochannel links due to the large XPD values, resulting in the worst $\mathrm{BER}$ performance. When XPD values are $\mathrm{XPD}_{\mathrm{LoS}}$ $=14 \mathrm{~dB}$ and $\mathrm{XPD}_{\mathrm{NLoS}}=5.8 \mathrm{~dB}$, the simulation results show a $2 \mathrm{~dB}$ gain, a $1.5 \mathrm{~dB}$ gain, and a $2 \mathrm{~dB}$ loss compared to the " $1 * 1$ " case and XPD values of $100 \mathrm{~dB}$ and $0 \mathrm{~dB}$, respectively, at the target BER of $10^{-3}$. In other words, the BER curve in this case is located between the curves for the upper and lower bounds.

Figures 7 and 8 show the BER and packet-error-rate (PER) performances when adopting the aforementioned combining techniques for the $3 \mathrm{D}$ multipolarized multipath Rician fading channel. The simulation scenario designated "Single" uses only a vertical dipole antenna at the BS and MS. The simulation scenarios "Dual EGC" and "Dual MRC" mean that a dual-polarized antenna is used at the Tx and Rx sides employing EGC and MRC combining techniques, respectively, and "Triple EGC" and "Triple MRC" both use triple-polarized antennas. As shown in Figures 7 and 8, it is obvious that the systems employing the triple-polarized antennas always show the best performance. The "Triple MRC BER" and "Triple EGC BER" provide $28 \mathrm{~dB}$ gain and $6 \mathrm{~dB}$ gain, respectively, compared to the "Single BER" at the target BER of $10^{-4}$. In addition, the relationship among the PER curves is similar to that found for the BER curves. Multipolarized systems using MRC provide more gain than those using EGC; the increased gain by the MRC with a 1-tap time-domain equalizer comes from the meaningful contribution of the LoS component in the Rician channel. In other words, what the MRC does better is that it is the optimum combining in terms of SNR and weights the strong antenna elements more than the weak. Figure 9 shows the throughput of five different simulation scenarios using the 3D multipolarized multipath Rician fading channel. At Eb/N0 of $12 \mathrm{~dB}$, for example, the "Triple MRC" scenario has a 20 times higher throughput than the "Single" scenario, and more than a 1.3 times higher throughput than the "Dual MRC" scenario.

The proposed channel model in [9] is verified by geometrically deriving the XPD value and then comparing it with the measured results. The verification results given in [9] validate the accuracy of the proposed channel model since the derived XPD values approximate the measured results. However, due to the modeling complexity in [9], it is difficult to be extended to support the cases with triplepolarized system as well as antenna array. In this paper, we verify our proposed channel model by directly specifying the XPD value according to the existing literatures and then

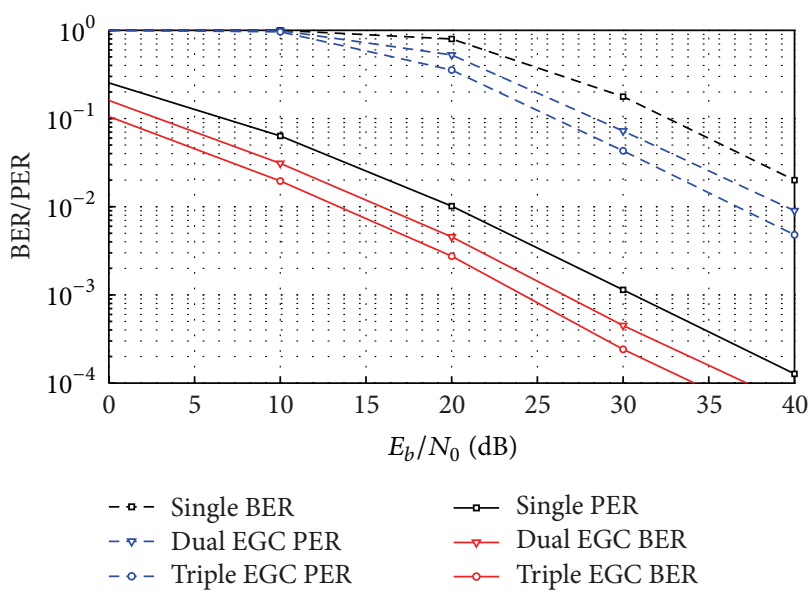

FIgUre 7: The BER and PER performances using EGC in the PS 3D multipolarized multipath Rician fading channel.

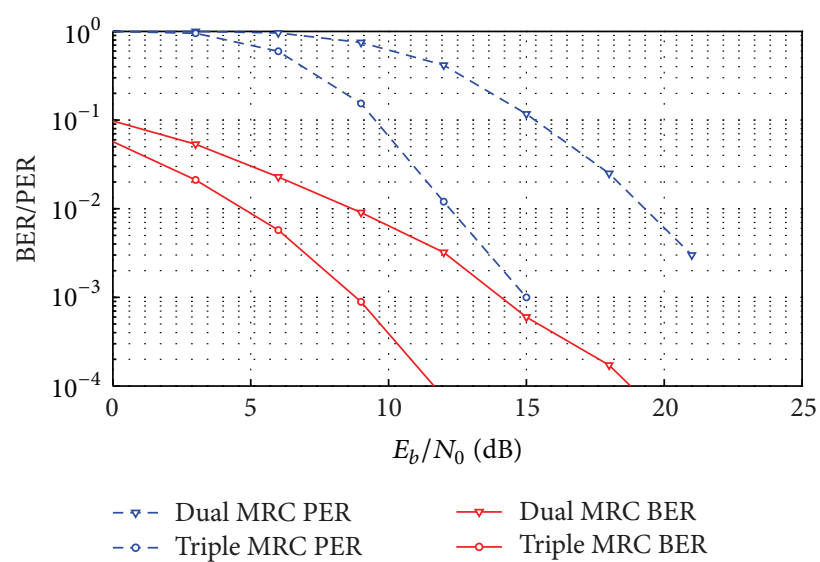

FIGURE 8: The BER and PER performances using MRC in the PS 3D multipolarized multipath Rician fading channel.

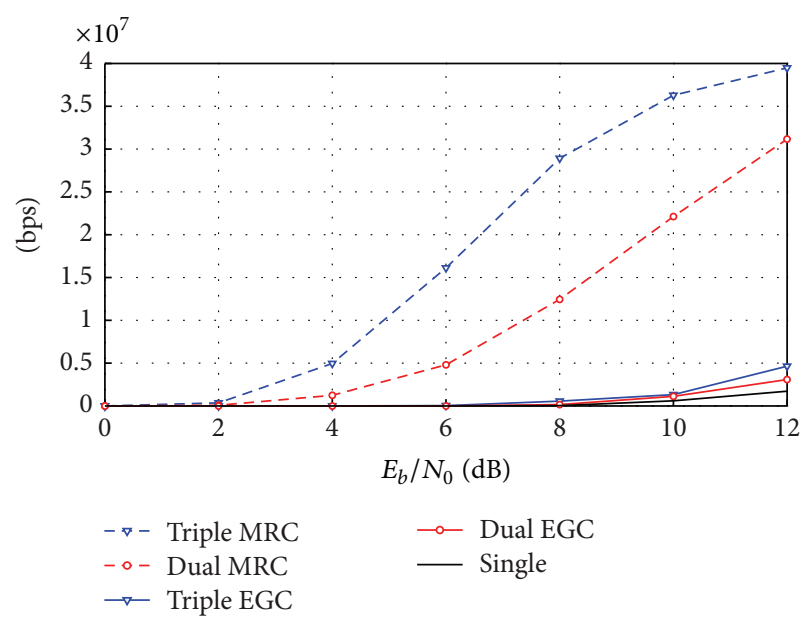

FIGURE 9: The throughput in the PS 3D multipolarized multipath Rician fading channel. 
test the proposed channel via the common diversity schemes. Based on the simulated BER/PER and throughput analysis by the diversity techniques, we also prove that the proposed channel model is accurate and reliable to be implemented for the computer simulation.

\section{Conclusions}

In this paper, PS 2D and 3D multipolarized multipath fading channel models have been presented based on the polarized characteristic of the SCM-E channel model. In order to verify and evaluate our proposed channel models, the measured $\mathrm{XPD}$ values in practical environments are employed. The BER and PER performances and the throughput using EGC and MRC combining techniques for multipolarized antenna systems have been presented. Under the polarized Rician fading channel, the triple-polarized antenna system employing MRC provides 20 times and 1.3 times higher throughput than a single antenna system and a dual-polarized antenna system with MRC, respectively, at an Eb/N0 of $12 \mathrm{~dB}$. In other words, the use of polarized antennas can greatly increase the reliability of wireless links, which can be turned into throughput increase, depending on the discrimination capabilities of the cross-channels of the polarized system. The use of a polarized antenna is also an efficient way to reduce antenna spacing, which helps to decrease both the size and the cost of a BS with a MIMO configuration.

\section{Conflict of Interests}

The authors declare that there is no conflict of interests regarding the publication of this paper.

\section{Acknowledgments}

This work was supported by Basic Science Research Program through the National Research Foundation of Korea (NRF) funded by the Ministry of Education (2013R1A1A2010287) and the project "Development of marine RF based ad hoc network for ship" from Ministry of Oceans and Fisheries (MOF), Korea. This work was also supported by Inha University Research grant. Part of this work has been published with preliminary form in the proceeding of the International Conference on Information Networking (ICOIN) in February 2012.

\section{References}

[1] M. A. Jensen and J. W. Wallace, "A review of antennas and propagation for MIMO wireless communications," IEEE Transactions on Antennas and Propagation, vol. 52, no. 11, pp. 2810$2824,2004$.

[2] O. Franek, J. B. Andersen, and G. F. Pedersen, "Diffuse scattering model of indoor wideband propagation," IEEE Transactions on Antennas and Propagation, vol. 59, no. 8, pp. 3006-3012, 2011.

[3] R. S. Blum, "Limiting case of a lack of rich scattering environment for MIMO radar diversity," IEEE Signal Processing Letters, vol. 16, no. 10, pp. 901-904, 2009.
[4] G. J. Foschini and M. J. Gans, "On limits of wireless communication in a fading environment when using multiple antennas," Wireless Personal Communications, vol. 6, no. 3, pp. 311-335, 1998.

[5] M. R. Andrews, P. P. Mitra, and R. Decarvalho, "Tripling the capacity of wireless communications using electromagnetic polarization," Nature, vol. 409, no. 6818, pp. 316-318, 2001.

[6] R. G. Vaughan, "Polarization diversity in mobile communications," IEEE Transactions on Vehicular Technology, vol. 39, no. 3, pp. 177-186, 1990.

[7] A. S. Y. Poon and D. N. C. Tse, "Degree-of-freedom gain from using polarimetric antenna elements," IEEE Transactions on Information Theory, vol. 57, no. 9, pp. 5695-5709, 2011.

[8] 3GPP, Spatial Channel Model for Multiple Input Multiple Output MIMO Simulations, vol. TR25.996, v6.1.0, September 2003.

[9] S.-C. Kwon and G. L. Stüber, "Geometrical theory of channel depolarization," IEEE Transactions on Vehicular Technology, vol. 60, no. 8, pp. 3542-3556, 2011.

[10] M. Shafi, M. Zhang, A. L. Moustakas et al., "Polarized MIMO channels in 3-D: models, measurements and mutual information," IEEE Journal on Selected Areas in Communications, vol. 24, no. 3, pp. 514-527, 2006.

[11] K. H. Jeon, B. Hui, K. H. Chang, H. S. Park, and Y. O. Park, "SISO polarized flat fading channel modeling for dual-polarized antenna systems," in Proceedings of the 26th International Conference on Information Networking (ICOIN '12), pp. 368373, February 2012.

[12] R. U. Nabar, H. Bölcskei, V. Erceg, D. Gesbert, and A. J. Paulraj, "Performance of multiantenna signaling techniques in the presence of polarization diversity," IEEE Transactions on Signal Processing, vol. 50, no. 10, pp. 2553-2562, 2002.

[13] ITU-R Rec. ITU-R M.1225, Guidelines for Evaluation of Radio Transmission Technologies for IMT-2000, 1997.

[14] J. J. A. Lempiäinen and J. K. Laiho-Steffens, “The performance of polarization diversity schemes at a base station in small/micro cells at $1800 \mathrm{MHz}$," IEEE Transactions on Vehicular Technology, vol. 47, no. 3, pp. 1087-1092, 1998.

[15] A. S. Lioumpas, G. K. Karagiannidis, and A. C. Iossifides, "Channel Quality Estimation Index (CQEI): a long-term performance metric for fading channels and an application in EGC receivers," IEEE Transactions on Wireless Communications, vol. 6, no. 9, pp. 3315-3323, 2007.

[16] A. Nasri, R. Schober, and M. Uysal, "Enhanced MRC for decode-and-forward cooperative diversity systems," IEEE Transactions on Wireless Communications, vol. 11, no. 10, pp. 3418-3423, 2012. 

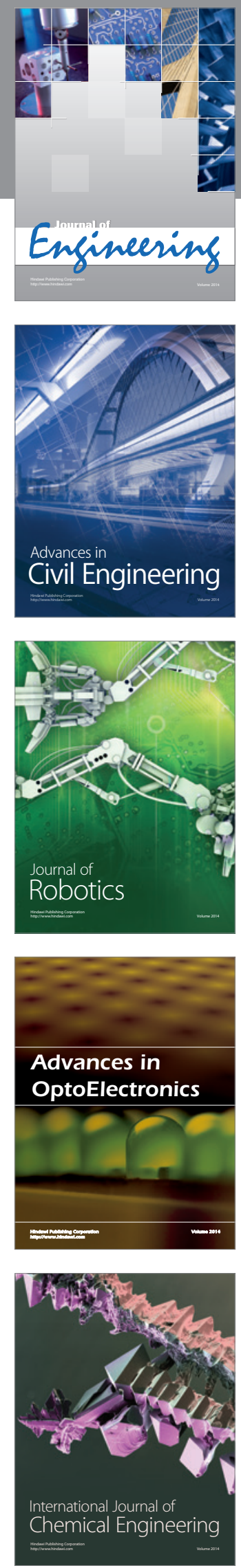

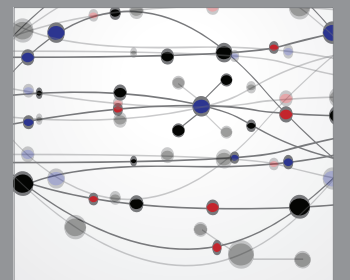

The Scientific World Journal
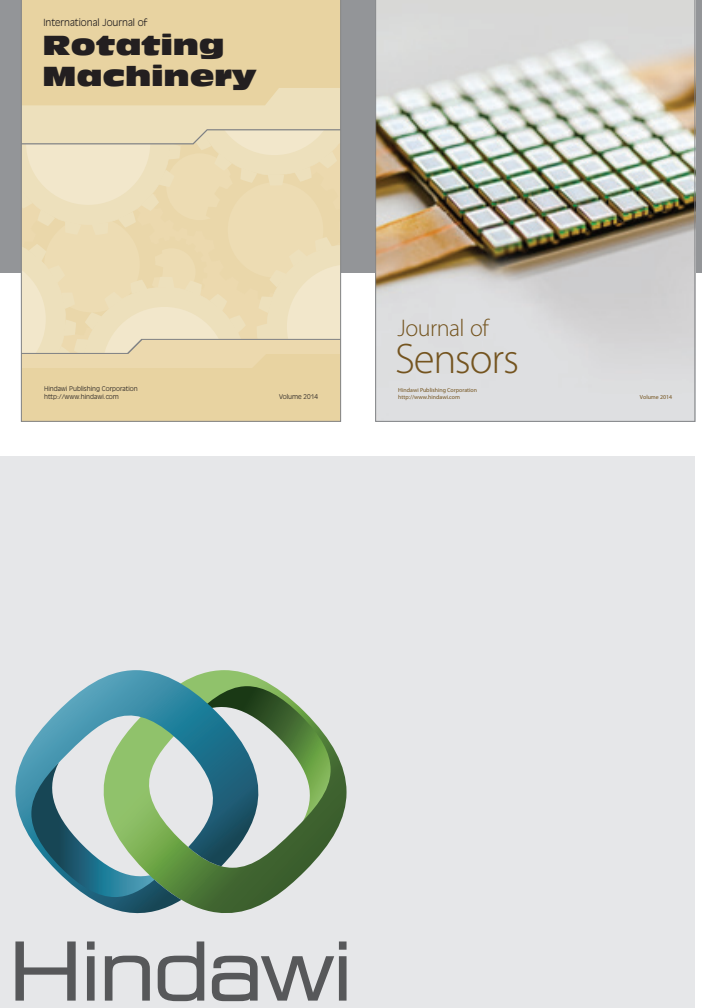

Submit your manuscripts at http://www.hindawi.com
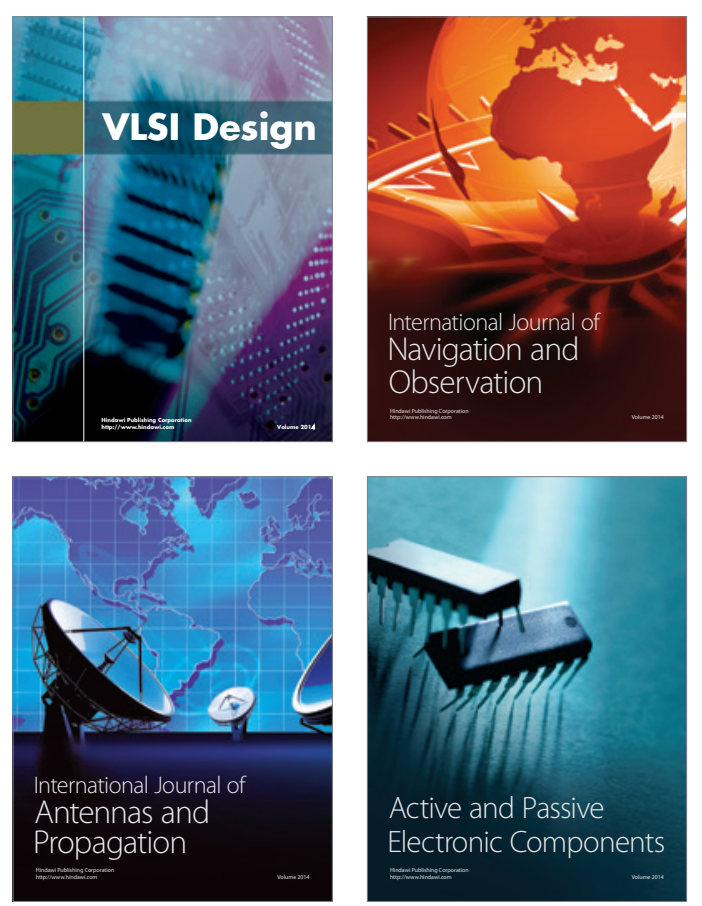
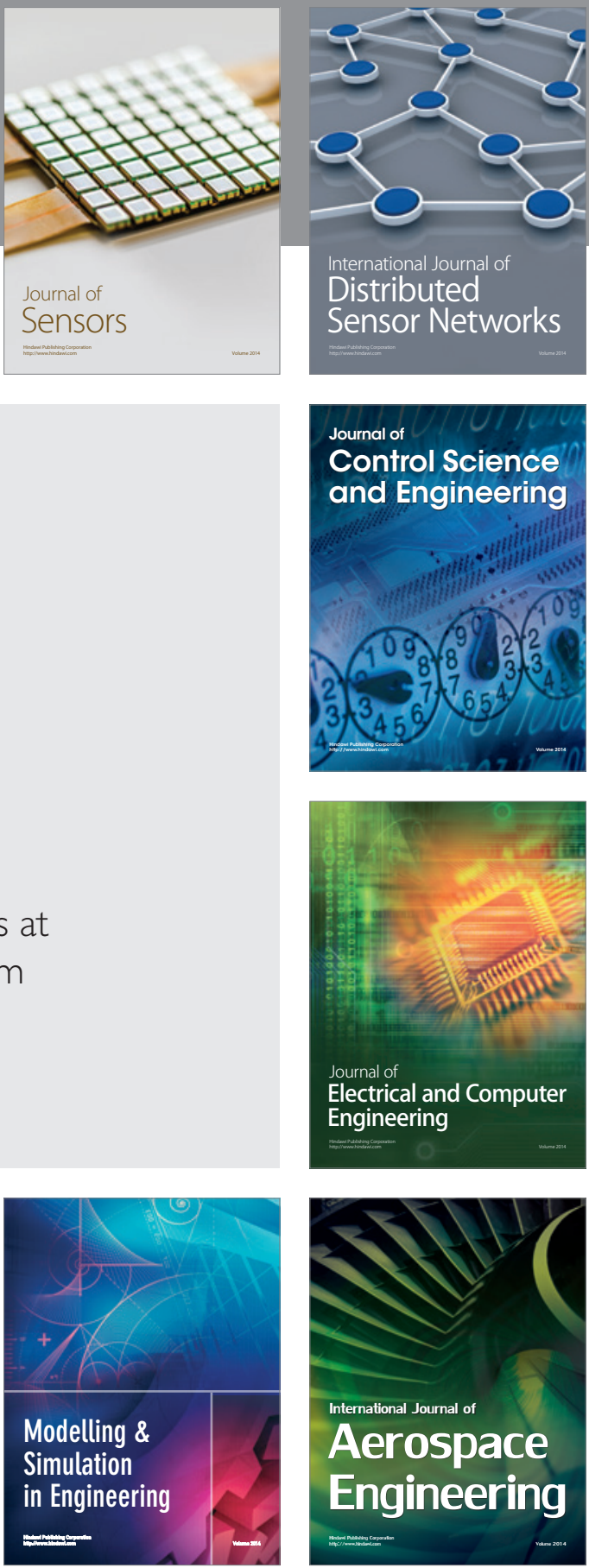

Journal of

Control Science

and Engineering
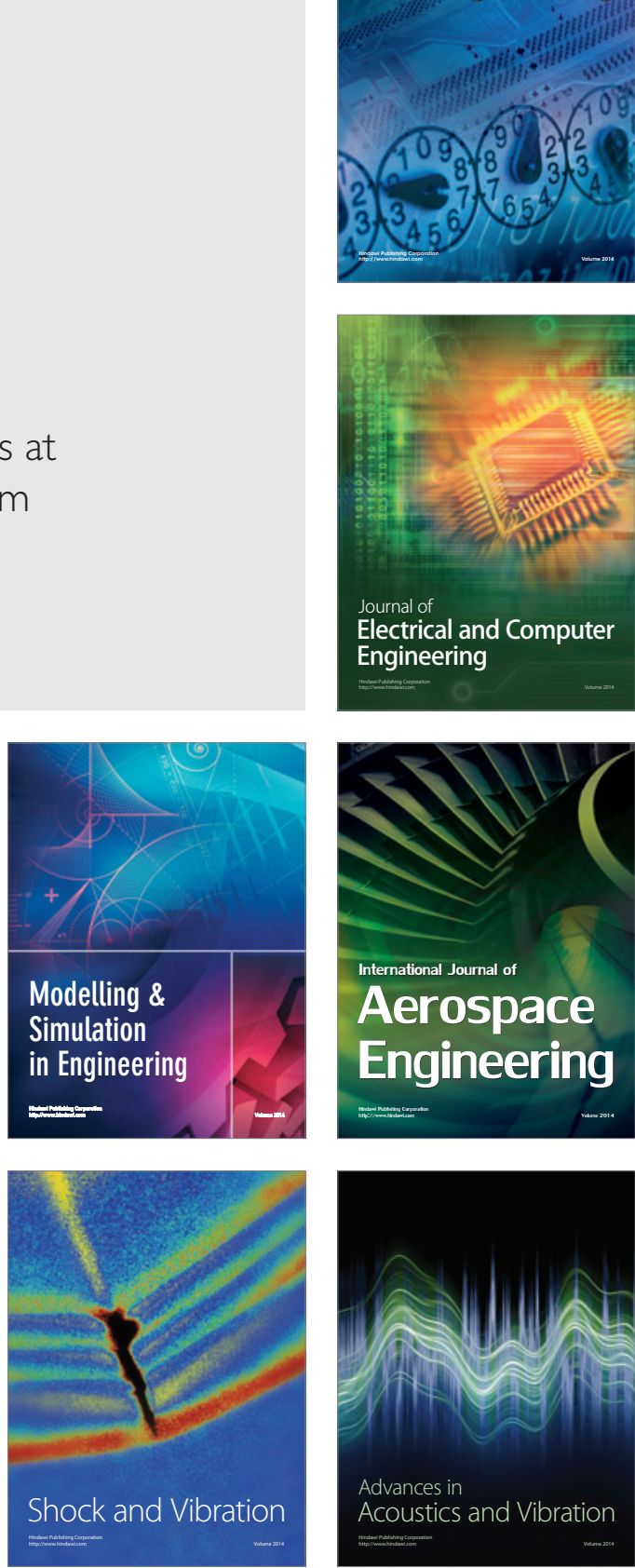